\title{
Serum vitamin $d$ level in iranian multiple sclerosis patients and their siblings: a case-control study
}

\begin{abstract}
Objectives: To measure serum vitamin D level in multiple sclerosis patients (MS) patients and their healthy siblings.

Methods: During the 12 month period (May 2014 - May 2015) in a case-control study, relapsing-remitting MS patients (based on McDonald 2005 criteria) and their healthy siblings were recruited. All participants were residents of Tehran city. Exclusion criteria were: 1 . consumption of drugs with known interaction with vitamin $\mathrm{D}$ metabolism during past two months 2. Current consumption of vitamin D and calcium supplements 3. Smoking 4. Medical disease which interferes with vitamin D metabolism. 5. Residents of other cities due to different latitude and sunshine expousure. Vitamin D levels (25 Hydroxy vitamin D3) were measured by ELISA (Enzyme Linked Immune Sorbent Assay) method. To reduce variability, all samples were analysed in batched assays. Differences in vitamin D levels between patients and controls were analysed.
\end{abstract}

Results: We evaluated 31 MS patients and 31 healthy siblings. The mean vitamin D level in the cases and controls was $12.7 \pm 5.5 \mathrm{ng} / \mathrm{ml}$ and $20.9 \pm 8.1 \mathrm{ng} / \mathrm{ml}$, respectively. There was a significant difference in serum vitamin $\mathrm{D}$ levels between cases and controls $(\mathrm{P}<0.001)$

Conclusion: In the present study Serum vitamin D levels were significantly lower in MS patients compared to that of healthy siblings. Nevertheless further research is needed to recommend routine vitamin D supplementation in MS patients.
Volume 6 Issue I - 2017

\author{
Asadollahi Marjan,' Ramezani Mahtab,' \\ Zarrinnezhad Neda,' Karimialavijeh Ehsan ${ }^{2}$ \\ 'Department of Neurology, Shahid Beheshti University of \\ Medical Sciences, Iran \\ ${ }^{2}$ Department of Emergency Medicine, Tehran University of \\ Medical Sciences, Iran
}

Correspondence: Ehsan Karimialavijeh, Tehran University of Medical sciences, Department of Emergency medicine, Shariati Hospital,Kargar Ave, Tehran, Iran, PO Box: 14| 17-13 |37; Tel 982161192240, Fax 9.82167E+1I,

Email e-karimi@sina.tums.ac.ir

Received: August 10, 2016 | Published: January 19, 2017

Keywords: vitamin d, multiple sclerosis, relapsing- remitting

Abbreviations: MS, multiple sclerosis; EBV, epstein-barr virus

\section{Introduction}

Multiple sclerosis (MS) is a chronic disease of central nervous system. This multifactorial Illness is one of the most common causes of disability among young adults. Genetic and environmental factors are Two major risk factors have been identified for the development of MS disease. ${ }^{1}$ Vitamin D deficiency, previous infection with EpsteinBarr virus (EBV) and smoking are the three recognized environmental risk factors for MS disease. ${ }^{2-5}$

Vitamin D deficiency has been recently identified as a potential risk factor for the development of MS disease. ${ }^{6}$ Vitamin D is a steroidal hormone which continuously transforms to 1,25 dihydroxy vitamin D3 or calcitriol, in the skin, liver and kidneys. Apart from its role in calcium metabolism, vitamin D has anti- inflammatory and anti- proliferative activities. It also has modulatory effects on the neurotrophins, growth factors and neurotransmitters in the nervous system. $^{7-9}$

Major sources of Vitamin D are food and sunshine. Recent studies have shown that even in the countries in which seafood is popular (that contains high amount of vitamin D), the amount of vitamin D provided by food rarely reaches 100 units per day. ${ }^{10}$ Sunshine remains the main natural source of vitamin D, provides approximately $90 \%$ of the body requirements. Although 15 to 30 minutes sunbathing can provide $10,000-20,000 \mathrm{IU}$ of vitamin $\mathrm{D}$, this supply disappears within a few weeks and cannot be replenished all year long, except in the tropical countries. ${ }^{11}$ So Vitamin D supplementation seems to be useful for people who have a vitamin D deficiency.

Prior studies reported low vitamin D level in Iranian multiple sclerosis patients. ${ }^{12,13}$ All of these reports have compared MS patients with healthy participants, but they have not considered the role of genetic factors in the development of MS disease. To eliminate the role of genetic factors, we have conducted the present study to measure serum vitamin D level in MS patients and their healthy siblings.

\section{Materials and methods}

During the 12 month period (May 2014 - May 2015, sampling took during a whole year to eliminate the seasonal variation in sunshine exposure) in a case-control study, relapsing-remitting multiple sclerosis patients who were attending the neurology clinic of an urban tertiary care university affiliated hospital, were recruited.

Eligibility criteria defined as definite relapsing-remitting multiple sclerosis (based on 2005 Mc Donald criteria, History, physical examination, BrainMRI, lumbarpuncture.) andlocalresidencyincapital city (Tehran). exclusion criteria were: 1 - concurrent consumption of drugs with known interaction with vitamin $\mathrm{D}$ metabolism during past two months(Aluminium hydroxide, Chlorothiazide, Chlorthalidone, Cholestyramine, Colesevelam, Colestipol, Digoxin, Fosphenytoin, Phenytoin, Phenobarbital, Primidone, Sucralfate, Orlistat, Minearl Oil, Metolazone, Methylclothyazide, Indapamide, Magnesium citrate and Magnesium hydroxide) 2- Usage of vitamin D and calcium supplements, 3- Smoking, 4- Patients with medical disease which interferes with vitamin D metabolism such as chronic kidney disease and parathyroid disease.5- residents of other cities were also excluded because the latitude of cities are different and this might has effects on sunshine exposure.

Initially patients' age, sex, disease pattern and Their EDSS (Expanded Disability Scale Score) were recorded. Controls were selected from healthy siblings of the patients. With the same method, their age and sex were recorded. Vitamin D levels (25 hydroxy vitamin 
D3) were measured by ELISA (Enzyme Linked Immune Sorbent Assay) method. To reduce variability, all samples were analyzed in batched assays.

Based upon current data the risk of extra-skeletal disorders (autoimmune, infectious, cancer and cardiovascular diseases) is higher when 25-hydroxyvitamin D levels are less than $20 \mathrm{ng} / \mathrm{ml} .{ }^{14}$ we have considered vitamin D levels under $20 \mathrm{ng} / \mathrm{ml}$ as vitamin D deficiency. Informed consent was obtained from all participants. The ethics committee of Shahid Beheshti University of medical sciences approved the conduct of the study. Data were analyzed using the statistical software SPSS 18 (SPSS, Chicago, IL). Differences in Vitamin D level between patients and controls were determined by sample $\square$-test. P value below 0.05 was considered significant.

\section{Results and discussion}

$31 \mathrm{MS}$ patients and 31 healthy first degree relatives of them were evaluated. There were 8 males and 23 females in the cases and the controls ( $25.8 \%$ and $74.2 \%$ respectively). The mean age of the cases and the controls was $30.9 \pm 11.4$ and $30.4 \pm 10.6$ years, respectively. From the statistical point of view, both case and control group members were age and sex matches. The mean EDSS in the patients was $1.5 \pm 1.05$ (Range 0-5.5). EDSS was 0 in 180 patients (58\%), between 1 and 2 in 60 patients (19\%) and greater than 2 in 70 patients $(23 \%)$.

The mean vitamin D level in the cases and controls was $12.7 \pm$ $5.5 \mathrm{ng} / \mathrm{ml}$ and $20.9 \pm 8.1 \mathrm{ng} / \mathrm{ml}$, respectively. There was a significant difference in serum vitamin $\mathrm{D}$ levels between these two groups $(\mathrm{P}<$ 0.001 ) (Table 1). The mean vitamin D level in patients with EDSS of 0 was $14 \pm 5.6 \mathrm{ng} / \mathrm{ml}, 8.2 \pm 3.7 \mathrm{ng} / \mathrm{ml}$ in patients with EDSS $1-2$ and $13.1 \pm 4.9 \mathrm{ng} / \mathrm{ml}$ in patients with EDSS more than 2. The difference of serum vitamin $\mathrm{D}$ levels among the mentioned groups with different EDSS was not statistically significant $(\mathrm{P}=0.07)$. Several studies have shown vitamin D promotes a $\mathrm{T}$ cell shift from Th1 to Th2, and limits the potential tissue damage associated with Th1 cellular immune responses. ${ }^{15,16}$ Vitamin D can also enhance the phagocytic function of monocytes and decrease the secretion of IL6, IL2, TNF-alpha and PGE2 by monocytes. ${ }^{17,18}$ Wergeland $\mathrm{S}$ et al., ${ }^{19}$ reported that vitamin $\mathrm{D}$ can decrease the demyelination process by a mechanism other than leukocyte infiltration in the CNS. ${ }^{19}$

Table I Vitamin D level in MS patients and their healthy first degree relatives

\begin{tabular}{lll}
\hline & \multicolumn{2}{l}{ MS Patients Controls } \\
\hline Mean Age(year) & $30.9 \pm 1.4$ & $30.4 \pm 1.6$ \\
Male (\%) & $8(25.8)$ & $8(25.8)$ \\
Female $(\%)$ & $23(74.19)$ & $23(74.19)$ \\
Mean Vitamin D level $(\mathrm{ng} / \mathrm{ml})$ & $12.7 \pm 5.5$ & $20.9 \pm 8.1$ \\
\hline
\end{tabular}

Several experimental studies on animals showed that vitamin $\mathrm{D}$ has a protective effect against induced EAE (an animal model of MS). Moreover, symptoms of EAE significantly improved after administration of vitamin D. These animal studies concluded that vitamin D has both preventive and curative effects in EAE. ${ }^{6}$

To our knowledge, there is only one study that directly analysed the risk of development of MS disease based on the serum level of $25(\mathrm{OH})$ vitamin D. Independent of geographic latitude and the degree of sun exposure, this study has showed a direct correlation between low levels of serum vitamin D and the development of MS disease. ${ }^{20}$

In a Finnish study, serum vitamin D levels of 40 patients with newly diagnosed MS (mean EDSS of 1.5) were compared to a healthy control group. Serum vitamin D levels were significantly lower in MS patients during the summer compared to normal subjects $(55 \mathrm{nmol} / 1$ and $80 \mathrm{nmol} / \mathrm{l}$, respectively). In contrast, during the winter months both groups had low serum vitamin D levels without any significant difference ${ }^{2}$. It seems that such low levels of vitamin D during winter have devastating effects on the members of both groups.

In Charles Pierrot-Deseilligny study, conducted in Paris, serum vitamin D level was measured in 167 relapsing-remitting MS patients. In $83 \%$ of these patients, serum vitamin D levels were in the insufficiency range (between25-75 nmol/l). In the remaining 17\% serum vitamin D levels were within deficiency range (below 25 $\mathrm{nmol} / \mathrm{l}){ }^{6}$ In Ascheiro A et al., ${ }^{3}$ study, it was showed that MS incidence can be reduced by $3 / 4$ by maintenance of serum vitamin $D$ level above $100 \mathrm{nmol} / 1$ during childhood and adolescence. ${ }^{3}$

In Goldberg P et al., ${ }^{21}$ Study MS attacks were reduced by $60 \%$ with administration of $5000 \mathrm{U} /$ day vitamin D for a two- year period. In a similar study, MS attacks were reduced by $50 \%$ with administration of $100 \mathrm{U} /$ day vitamin D for 48 weeks. These two studies had no control group..$^{21,22}$ The results of a large prospective study on a group of relapsing-remitting MS patients, with different disease durations, showed that an increment of $10 \mathrm{nmol} / 1$ in serum vitamin D level could reduce the prevalence of MS attacks by $9-12 \%,{ }^{23}$

In the present study controls were not selected randomly from the community. To decrease the effect of genetic factors, the controls were selected from the patients' healthy siblings. To reduce the seasonal variation in sunshine exposure we recruited participants during a whole year. All participants were residents of a same city (Tehran). Considering these strict criteria our results more clearly shows the role of low vitamin D in MS patients.

\section{Conclusion}

In the present study, serum vitamin D level in MS patients was significantly lower than their siblings ( $\mathrm{P}$ value $<0.001$ ). This finding is compatible with the results of other similar studies. Nevertheless more research is needed to recommend routine vitamin D supplementation in MS patients.

\section{Acknowledgments}

None.

\section{Conflicts of interest}

None.

\section{Funding}

None.

\section{References}

1. Ebers GC. Environmental factors and multiple sclerosis. Lancet Neurol. 2008;7(3):268-277.

2. Ascherio A, Munger K. Epidemiology of multiple sclerosis: from risk factors to prevention. Seminars in neurology. 2008;28(1):17-28.

3. Ascherio A, Munger KL. Environmental risk factors for multiple sclerosis. Part I: the role of infection. Ann Neurol. 2007;61(4):288-299.

4. Giovannoni G, Ebers G. Multiple sclerosis: the environment and causation. Curr Opin Neurol. 2007;20(3):261-268.

5. Holmoy T, Hestvik ALK. Multiple sclerosis: immunopathogenesis and controversies in defining the cause. Curr Opin Infect Dis. 2008;21(3):271-278. 
6. Pierrot-Deseilligny C. Clinical implications of a possible role of vitamin D in multiple sclerosis. $J$ Neurol. 2009;256(9):1468-1479.

7. Chaudhuri A. Why we should offer routine vitamin D supplementation in pregnancy and childhood to prevent multiple sclerosis. Medical hypotheses. 2005;64(3):608-618.

8. Heaney RP, Davies KM, Chen TC, et al. Human serum 25-hydroxycholecalciferol response to extended oral dosing with cholecalciferol. Am J Clin Nutr. 2003;77(1):204-210.

9. Vieth R. What is the optimal vitamin D status for health? Prog Biophys Mol Bio. 2006;92(1):26-32.

10. Mark BL, Carson JAS. Vitamin D and autoimmune disease - Implications for practice from the multiple sclerosis literature. $J$ Am Diet Assoc. 2006;106(3):418-424.

11. Vieth R. Vitamin D toxicity, policy, and science. $J$ Bone Miner Res. 2007;22:V64-V8

12. Mansouri B, Asadollahi S, Heidari K, et al. Risk factors for increased multiple sclerosis susceptibility in the Iranian population. $J$ Clin Neurosci. 2014;21(12):2207-2211.

13. Shaygannejad V, Golabchi K, Haghighi S, et al. A Comparative Study of $25(\mathrm{OH})$ Vitamin D Serum Levels in Patients with Multiple Sclerosis and Control Group in Isfahan, Iran. International journal of preventive medicine. 2010;1(3):195-201.

14. Bouillon R, Van Schoor NM, Gielen E, et al. Optimal vitamin D status: a critical analysis on the basis of evidence-based medicine. J Clin Endocrinol Metab. 2013;98(8):E1283-1304.

15. Boonstra A, Barrat FJ, Crain C, et al. 1 alpha,25-dihydroxyvitamin D3 has a direct effect on naive CD4(+) T cells to enhance the development of Th2 cells. J Immunol. 2001;167(9):4974-4980.
16. Overbergh L, Decallonne B, Waer M, et al. 1alpha,25-dihydroxyvitamin D3 induces an autoantigen-specific T-helper 1/T-helper 2 immune shift in NOD mice immunized with GAD65 (p524-543). Diabetes. 2000;49(8):1301-1307.

17. D'Ambrosio D, Cippitelli M, Cocciolo MG, et al. Inhibition of IL-12 production by 1,25-dihydroxyvitamin D3. Involvement of NF-kappaB downregulation in transcriptional repression of the p40 gene. The Journal of clinical investigation. 1998;101(1):252-262.

18. Xu H, Soruri A, Gieseler RKH, et al. 1,25-Dihydroxyvitamin-D3 Exerts Opposing Effects to Il-4 on Mhc Class-Ii Antigen Expression, Accessory Activity, and Phagocytosis of Human Monocytes. Scand J Immunol. 1993;38(6):535-540.

19. Wergeland S, Torkildsen O, Myhr KM, et al. Dietary vitamin D3 supplements reduce demyelination in the cuprizone model. PloS one. 2011;6(10):e26262.

20. Munger KL, Levin LI, Hollis BW, et al. Serum 25-hydroxyvitamin D levels and risk of multiple sclerosis. Jama. 2006;296(23):2832-2838.

21. Goldberg P, Fleming MC, Picard EH. Multiple sclerosis: decreased relapse rate through dietary supplementation with calcium, magnesium and vitamin D. Medical hypotheses. 1986;1(2):193-200.

22. Wingerchuk DM, Lesaux J, Rice GPA, et al. A pilot study of oral calcitriol (1,25-dihydroxyvitamin D-3) for relapsing-remitting multiple sclerosis. J Neurol Neurosur PS. 2015;76(9):1294-1296.

23. Simpson S, Taylor B, Blizzard L, et al. Higher 25-hydroxyvitamin D Is Associated with Lower Relapse Risk in Multiple Sclerosis. Ann Neurol. 2010;68(2):193-203. 